\title{
Multiple Unmanned Aerial Vehicles Deployment and User Pairing for Non-Orthogonal Multiple Access Schemes
}

\author{
Jie Wang, Student Member, IEEE, Miao Liu, Member, IEEE, Jinlong Sun, Member, IEEE, \\ Guan Gui, Senior Member, IEEE, Haris Gacanin, Senior Member, IEEE, \\ Hikmet Sari, Fellow, IEEE, and Fumiyuki Adachi, Life Fellow, IEEE
}

\begin{abstract}
Non-orthogonal multiple access (NOMA) significantly improves the connectivity opportunities and enhances the spectrum efficiency (SE) in the fifth generation and beyond (B5G) wireless communications. Meanwhile, emerging B5G services demand of higher SE in the NOMA based wireless communications. However, traditional ground-to-ground (G2G) communications are hard to satisfy these demands, especially for the cellular uplinks. To solve these challenges, this paper proposes a multiple unmanned aerial vehicles (UAVs) aided uplink NOMA method. In detail, multiple hovering UAVs relay data for a part of ground users (GUs) and share the subchannels with the left GUs that communicate with the base station (BS) directly. Furthermore, this paper proposes a Kmeans clustering based UAV deployment and location based user pairing scheme to optimize the transceiver association for the multiple UAVs aided NOMA uplinks. Finally, a sum power minimization based resource allocation problem is formulated with the lowest quality of service $(\mathrm{QoS})$ constraints. We solve it with the message-passing algorithm and evaluate the superior performances of the proposed scheduling and paring schemes on SE and energy efficiency (EE). Extensive experiments are conducted to compare the performances of the proposed schemes with those of the single UAV aided NOMA uplinks, G2G based NOMA uplinks, and the proposed multiple UAVs aided uplinks with a random UAV deployment. Simulation results demonstrate that the proposed multiple UAVs deployment and user pairing based NOMA scheme significantly improves the EE and the SE of the cellular uplinks at the cost of only a little relaying power consumption of the UAVs.
\end{abstract}

Index Terms-Unmanned aerial vehicle (UAV), non-orthogonal multiple access, UAV deployment, user pairing, energy efficiency, spectrum efficiency.

\section{INTRODUCTION}

Ubiquitous connectivity with ultra-high spectrum efficiency and system throughput is one of the crucial requirements for

This work was supported by the Project Funded by the National Science and Technology Major Project of the Ministry of Science and Technology of China under Grant TC190A3WZ-2, the Jiangsu Specially Appointed Professor under Grant RK002STP16001, the Innovation and Entrepreneurship of Jiangsu High-level Talent under Grant CZ0010617002, the Six Top Talents Program of Jiangsu under Grant XYDXX-010, the 1311 Talent Plan of Nanjing University of Posts and Telecommunications. (Corresponding authors: Guan Gui.)

J. Wang, M. Liu, J. Sun, G. Gui and H. Sari are with the College of Telecommunications and Information Engineering, Nanjing University of Posts and Telecommunications, Nanjing 210003, China. (e-mails: \{2018010223, liumiao, sunjinlong, guiguan, hikmet $\} @$ njupt.edu.cn)

H. Gacanin is with the Nokia Bell Labs, 2018 Antwerp, Belgium (e-mail: harisg@ieee.org).

F. Adachi is with the Research Organization of Electrical Communication (ROEC) Tohoku University, Sendai 980-8577 Japan (e-mail: adachi@ecei.tohoku.ac.jp) the fifth generation and beyond (B5G) wireless communication systems [1]-[3] and internet of things (IoT) [4]-[7]. Through significantly improving the connectivity and spectrum efficiency [8]-[12], non-orthogonal multiple access (NOMA) has become a promising solution for the $\mathrm{B} 5 \mathrm{G}$ communications. Generally, in the NOMA aided systems, multiple users with different channel conditions are multiplexed in power domain and served on the same sub-channels simultaneously. Then the receivers utilize successive interference cancellation (SIC) technique to remove intra-channel interference with the decoding order based on channel conditions [11] $-[13]$. Especially in recent years, many other promising techniques have been widely researched through combining NOMA. For examples, multiple input multiple-output based NOMA techniques were investigated in [14], [15]. Dynamic resource allocation schemes for improving performances of the NOMA based networks were explored in [16]-[18]. Dynamic user grouping was explored in [19]. A new definition of fairness for NOMA was proposed in [20]. And also applying NOMA to heterogeneous networks were discussed in [21]-[23].

Although plenty of previous works have proved the excellent performances of NOMA on massive connectivity and high SE, there still exist many challenges for applying NOMA to support B5G communications, especially for designing effective uplink NOMA schemes [24]-[27]. Firstly, the spectrum efficiency (SE) of the cellular systems needs to be further improved by NOMA. Secondly, the energy efficiency (EE) of the cellular systems needs to be raised by NOMA. Because of the high mobility, flexible deployment and line-ofsight (LoS) channels of unmanned aerial vehicle (UAV), UAV aided B5G technologies have attracted increasing attentions from both academia, and industry [28]-[32]. Specifically, there are many existing works about integrating NOMA into UAV aided communication systems to improve the throughput of the systems, mainly focusing on optimal UAV deployment, optimal user paring strategy, optimal sub-channel allocation scheme and fair control for different users [33]-[35]. In [33], a mobile UAV based downlink NOMA model was considered, and then UAV trajectory design and resource allocation in NOMA are jointly optimized to maximize the capacity of the system. M. T. Nguyen et al. [34] also considered a single UAV based downlink NOMA system, and optimized the user pairing, power allocation, and UAV placement to maximize the minimum sum rate for individual 
user pair. The communication reliability and fairness among users for a single-UAV aided downlink NOMA cell system was investigated in [35] by optimizing the UAV's altitude and the users' power allocation coefficients. The maximum stable throughput of a single-UAV based uplink NOMA cell system was investigated by [26], in terms of the altitude and bandwidth of the UAV. R. Duan et al. [27 considered a multi-UAV aided IoT NOMA uplink transmission system and evaluated that combining NOMA and UAV communication can achieve higher capacity than orthogonal multiple access (OMA) based UAV communication systems.

To the best of our knowledge, utilizing multiple UAVs to improve the SE and the EE of the uplink NOMA cell systems have not been investigated. Motivated by this background, in this paper a multiple UAVs aided NOMA technology is proposed to enhance the SE and the EE of the cellular uplinks. Particularly, we propose a K-means clustering based UAV deployment scheme and a location based user pairing scheme. In addition, considering the energy limited GUs' devices, a sum power minimization based resource allocation scheme is adopted to evaluate the outperformance of the proposed schemes. The contributions of this paper can be summarized as follows:

- We propose a multiple UAVs aided NOMA technology to enhance the SE and the EE of the uplink cellular systems.

- A K-means clustering based UAV deployment scheme is proposed to optimize the serving areas of the proposed multiple UAVs aided uplink NOMA.

- A location based user pairing scheme is proposed to determine the decoding orders of the users for the proposed multiple UAVs aided uplink NOMA.

- To save transmitted power for the GUs' devices, we consider a sum power minimization based resource allocation scheme with the lowest quality of service (QoS) constraints to evaluate the performance of the proposed schemes for the multiple UAVs aided uplink NOMA.

\section{Multiple UAVs Aided Uplink NOMA Cellular SYSTEM MODEL}

A multiple UAVs aided uplink NOMA cellular system is consists of a BS, $N$ GUs and $M$ UAVs. The BS is located at the center of a ground cell of which the radius is $R$. And $N$ GUs are randomly and uniformly distributed in the coverage of the cell. For applying UAVs to improve the SE and EE of the uplink transmissions in this system, the proposed multiple UAVs aided uplink NOMA technology is designed as follows. Firstly, all the $M$ UAVs are considered as relays to help half of the GUs in hovering pattern. At the same time, the left GUs communicate with the BS directly. Especially, the relayed and the directly transmitting users can be paired together and their messages are accommodated on the same spectrums. In detail, the UAVs relay data by using decode-and-forward (DF) protocol and full duplex (FD) technology. Specially, each UAV is equipped with two antennas. One antenna receives signals and another antenna transmits signals simultaneously. For convenience, the receiving antenna and the transmitting antenna of each UAV are denoted as $a$ and $b$ respectively. To be general, the residual self-interference (RSI) after SIC in UAVs is considered, see Fig. 1 .

As is shown in Fig. 1 the relay links and direct links in same color are located on the same sub-channels. $\theta_{1}, \cdots, \theta_{m}, \cdots, \theta_{M}$ denote the elevation angle between the BS and the UAVs. $\varphi_{i, m}$ represents the elevation angle between the $i$-th GU and the $m$-th UAV. We assume that the total available frequency bands of the system are equally divided into $Z^{T}$ orthogonal sub-channels and the bandwidth of each sub-channel is $B_{0}$.

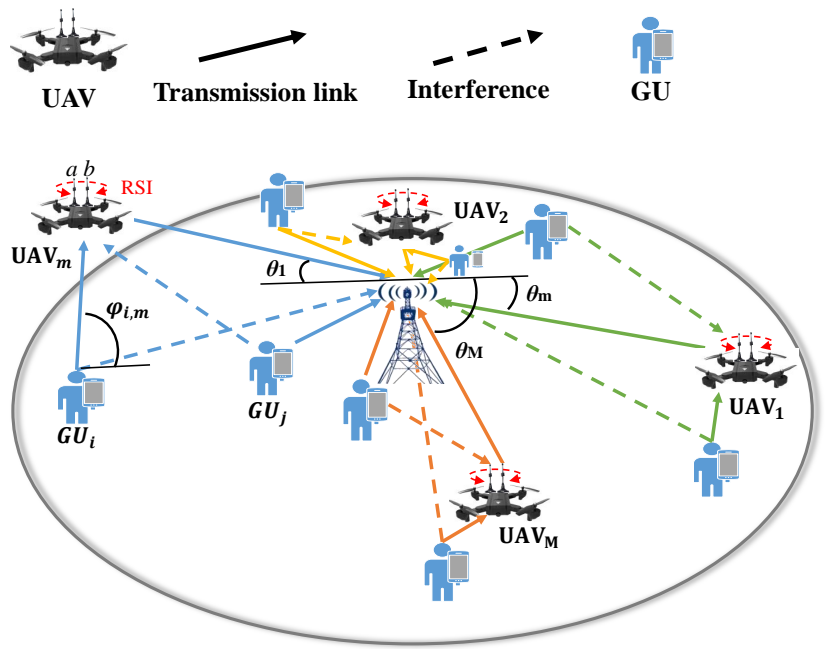

Fig. 1: The multiple UAVs aided uplink NOMA cellular system model.

In this model, let $G U_{n}$ denote the $n$-th GU, $n \in$ $\{1,2, \cdots, N\}$ and $U A V_{m}$ denote the $m$-th UAV, $m \in$ $\{1,2, \cdots, M\}$. The typical channel models in [36] for the ground to ground links and the ground to air links are adopted in this system model. In detail, the GU-BS channels are modeled as Rayleigh fading channels and the GU-UAV channels are LoS channels or NLoS channels depending on the environments. Specially, the UAV-BS channels in this system model are always LoS channels based on our UAV deployment scheme, which will be detailed in Section III The channels between receiving antenna $a$ and transmitting antenna $b$ are also modeled as LoS channels. Let $g_{n, m, z}^{U A}$ denote the channel gain between $G U_{n}$ and $U A V_{m}$ on sub-channel $z$, $z \in\left\{1,2, \cdots, Z^{T}\right\}, g_{n, m, z}^{A B}$ denote the channel gain between the $U A V_{m}$ and the $\mathrm{BS}$ on sub-channel $z$ for $G U_{n}, g_{n, B, z}^{U B}$ denote the channel gain between the $G U_{n}$ and the BS on subchannel $z$ and $g_{a, b, z}$ denotes the channel gain between the antenna $a$ and the antenna $b$ on sub-channel $z, P$ denotes the LoS probability of GU-UAV channels. Based on [36], they are defined as

$$
\begin{gathered}
g_{n, m, z}^{U A}=\left\{\begin{array}{c}
\eta_{L o S}\left(d_{n, m}^{U A}\right)^{-\alpha_{R}}, \quad \text { with } P \\
\eta_{N L o S}\left(d_{n, m}^{U A}\right)^{-\alpha_{R}}, \text { with } 1-P
\end{array}\right. \\
g_{n, m, z}^{A B}=\eta_{L o S}\left(d_{m, B}^{A B}\right)^{-\alpha_{R}} \\
g_{a, b, z}=\eta_{L o S}\left(d_{a, b}\right)^{-\alpha_{R}}
\end{gathered}
$$




$$
g_{n, B, z}^{U B}=\left(d_{n, B}^{U B}\right)^{-\alpha_{B}}\left|h_{z}\right|^{2}
$$

where $P=1 /\left[1+\psi \exp \left(-\beta\left(\varphi_{n, m}-\psi\right)\right)\right]$, in which $\varphi_{n, m}=$ $\left(180^{\circ} / \pi\right) \arcsin \left(H_{m} / d_{n, m}^{U A}\right)$ is elevation angle between the $G U_{n}$ and the $U A V_{m}, \beta$ and $\psi$ are environment dependent constant values. $H_{m}$ is the height of the $U A V_{m} . d_{n, m}^{U A}$, $d_{m, B}^{A B}, \quad d_{a, b}, d_{n, B}^{U B}$ denote the distance between $G U_{n}$ and $U A V_{m}$, the distance between $U A V_{m}$ and BS, the distance between receiving antenna $a$ and transmitting antenna $b$. The distance between $G U_{n}$ and BS, respectively. $\eta_{L o S}$ and $\eta_{N L O S}$ denote additional attenuation factors of LoS and NLoS channels, respectively. $\alpha_{R}$ and $\alpha_{B}$ denote the path loss factor of the ground to air channel and the Rayleigh fading channel, respectively. $h_{z}$ denotes the complex Gaussian channel coefficient which follows the distribution of $C N(0,1)$.

\section{The Proposed UAV Deployment Scheme}

The performance of the multiple UAVs aided uplink NOMA cellular system is determined by the UAV deployment. Aim to maximize the SE as well as the $\mathrm{EE}$ of the considered cellular uplinks, we propose a K-means clustering based UAV deployment scheme (Multi-UAV-K-cluster scheme).

We assume that all the GUs' locations are known to the UAVs and the BS. Thus, the GUs can be equally divided into two groups based on their Euclidean distance between GUs and BS. The central group and the edge group, respectively, are shown by the orange area and white area in Fig. 2 The GUs in orange area are closer to the BS, thus they are assigned to communicate with the BS directly and we denote them as direct GUs. Similarly, the GUs in the white area are far to the BS, thus they are relayed by the UAVs and we denote them as relay GUs. After determining the direct GUs and relay GUs, all the relay GUs can be clustered into $M$ clusters based on their locations by the BS with K-means clustering algorithm. In detail, to obtain a uniformly distributed clustering results, we choose the coordinates of the $M$ GUs that are closest to the $M$ points of $\Phi_{C}$ as the initial centroids. The $M$ points of $\Phi_{C}$ are uniformly distributed at the edge of the cell, see (5). Specially, the GU that is assigned as one centroid will be excluded in the following computing to avoid repeatedly assigning one $\mathrm{GU}$ as different centroids.

$$
\begin{aligned}
& \Phi_{C}=\{(\mathrm{R} \cos (2 \pi / M), R \sin (2 \pi / M)), \\
& (\mathrm{R} \cos (4 \pi / M), R \sin (4 \pi / M)), \cdots,(\mathrm{R} \cos (2 \pi), R \sin (2 \pi))\}
\end{aligned}
$$

Then, the $M$ UAVs are scheduled to hovering on the centroids of $M$ clusters with random heights. To resist the interference from direct GUs, the heights of all the UAVs should be adjusted until all the UAV-BS links have LoS channels. The detailed Multi-UAV-K-cluster scheme are summarized in Algorithm 1. After the UAV deployment, each relay GU will communicate with the UAV that is deployed above the cluster it belongs to.

\section{Sum Power Minimization Problem Formulation}

To save transmitted power for the GUs' devices, we consider a sum power minimization based resource allocation
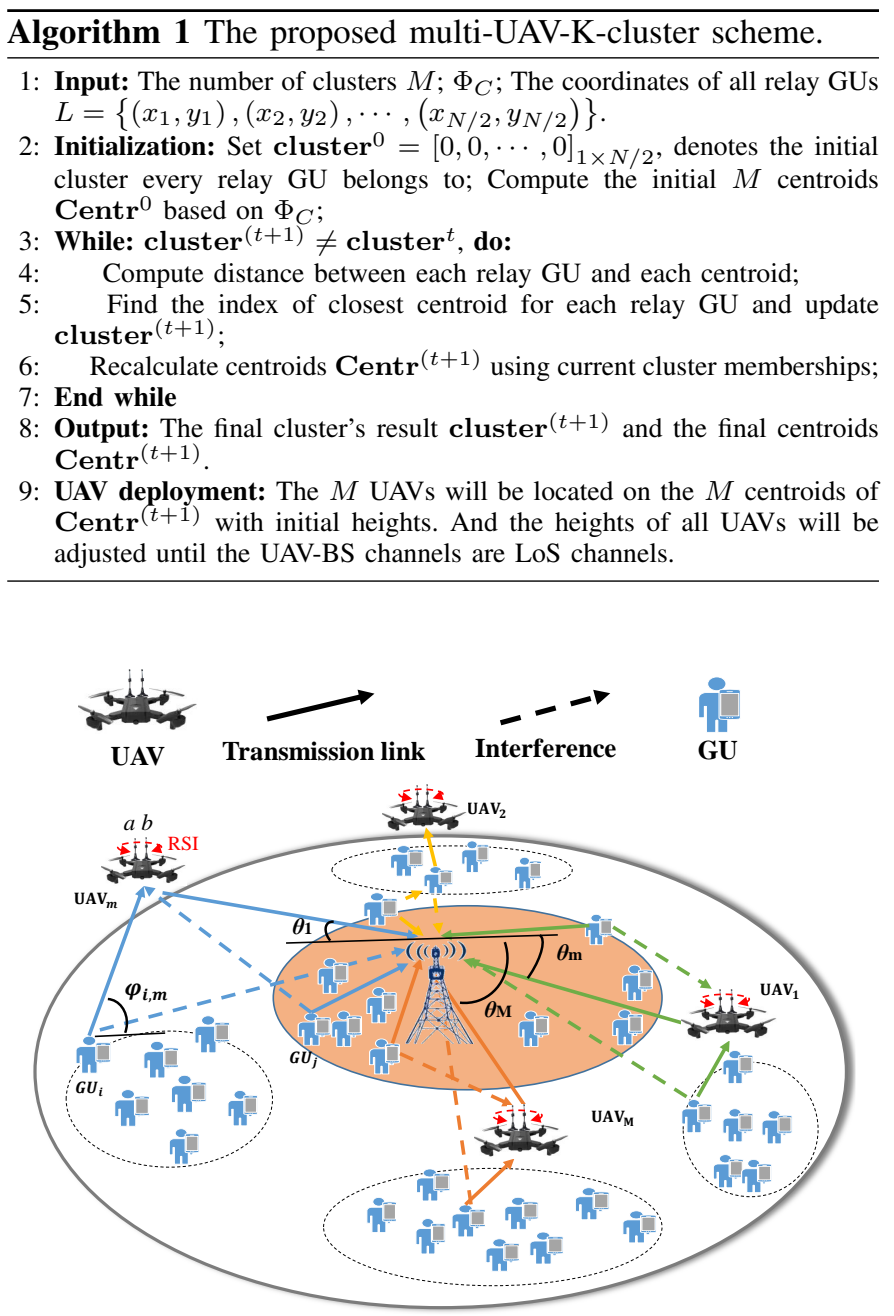

Fig. 2: The demonstration of the Multi-UAV-K-cluster scheme.

scheme with the lowest QoS constraints to evaluate the performance of the proposed schemes for the multiple UAVs aided uplink NOMA. For convenience to formulate the sum power minimization problem, we make an assumption as following.

Assumption 1: $G U_{i}$ and $G U_{j}$ are paired to assign different receivers and share the same sub-channel $z$, and $G U_{i}$ is assigned to $U A V_{m}$ while $G U_{j}$ is assigned to BS directly.

Since UAV-BS channels are LoS channel, they are obviously much better than the GU-BS channels. Therefore, the SINR of the UAVs relayed signals on the BS is higher than the directly transmitted signals. Accordingly, the BS is able to successfully decode the relayed data in the first order. As a result, under Assumption 1, the interference introduced to $G U_{j}$ only consists of Additive White Gaussian Noise (AWGN). Meanwhile, the interference introduced to $G U_{i}$ includes the co-channel interference (CI) from $G U_{j}$, the RSI of the $U A V_{m}$ and AWGN. Let $\gamma_{i, m, z}^{U A}$ denote the SINR from the $G U_{i}$ to $U A V_{m}$ on sub-channel $z, \gamma_{i, m, z}^{A B}$ denote the SINR from $U A V_{m}$ to the BS on sub-channel $z$ for relaying $G U_{i}$ 's data, and $\gamma_{j, B, z}^{U B}$ denote the SINR from $G U_{j}$ to the BS. Then, 
they are respectively computed as

$$
\begin{gathered}
\gamma_{i, m, z}^{U A}=\frac{\underbrace{N_{0} B_{0}}_{A W G N}+\underbrace{g_{j, m, z}^{U A} p_{j, B, z}^{U B}}_{C I}+\underbrace{U A}_{R S I} \underbrace{U g_{a, b, z} p_{i, m, z}^{A B}}_{i, m, z}}{\gamma_{i, m, z}^{A B}=\frac{g_{i, m, z}^{A B} p_{i, m, z}^{A B}+g_{i, B, z}^{U B} p_{i, m, z}^{U A}}{\underbrace{N_{0} B_{0}}_{A W G N}+\underbrace{g_{j, B, z}^{U B} p_{j, B, z}^{U B}}_{C I}}} \\
\gamma_{j, B, z}^{U B}=\frac{g_{j, B, z}^{U B} p_{j, B, z}^{U B}}{\underbrace{N_{0} B_{0}}_{A W G N}}
\end{gathered}
$$

where $p_{i, m, z}^{U A}, p_{i, m, z}^{A B}, p_{j, B, z}^{U B}$ are the transmitted power on subchannel $z$, consumed by $G U_{i}, U A V_{m}$, and $G U_{j}$ respectively. In addition, $N_{0}$ denotes the power spectral density of the AWGN, and $\tau$ denotes the RSI coefficient. Especially, $\gamma_{i, m, z}^{A B}$ also benefits from the signals of $G U_{i}$ received by the BS, which can be computed as $g_{i, B, z}^{U B} p_{i, m, z}^{U A}$. By denoting $R_{i, m, z}^{U A}$, $R_{i, m, z}^{A B}$ and $R_{j, B, z}^{U B}$ as the data rates of the $G U_{i}, U A V_{m}$ and $G U_{j}$ on sub-channel $z$, their upper bound can be further computed based on the Shannon's capacity.

We define a matrix $\mathbb{U}$ with a size of $N \times M$, in which the binary variable $u_{n, m} \in\{0,1\}$ represents whether the $G U_{n}$ is assigned to $U A V_{m}$. If $G U_{n}$ is assigned to $U A V_{m}$ for transmission, then $u_{n, m}=1$, otherwise $u_{n, m}=0$. Likewise, a matrix $\mathbb{S}$ with a size of $1 \times N$ is defined to denote which GUs are assigned to the BS. If $G U_{n}$ is scheduled to communicate with BS directly, then its binary assignment variable $s_{n, B}=1$, otherwise $s_{n, B}=0$. Besides, a matrix $\mathbb{Z}$ with a size of $N \times Z^{T}$ is defined to denote the sub-channel allocation results. Its binary element $v_{n, z} \in\{0,1\}$ denotes whether the sub-channel $z$ is allocated to $G U_{n}$. If sub-channel $z$ is allocated to $G U_{n}$, then $v_{n, z}=1$, otherwise $v_{n, z}=0$. Moreover, if $p_{n}^{U}, p_{m}^{A}$ denote the total transmitted power of $G U_{n}$ and $U A V_{m}$, then they can be computed as 97 and $(10)$. Similarly, let $R_{n}^{U}$ denotes the sum rate of $G U_{n}$, then it can be computed as [11,

$$
\begin{aligned}
& p_{n}^{U}= \sum_{z=1}^{Z^{T}} v_{n, z}\left(\left(\sum_{m=1}^{M} u_{n, m} p_{n, m, z}^{U A}\right)+s_{n, B} p_{n, B, z}^{U B}\right) \\
& p_{m}^{A}=\sum_{n=1}^{N} \sum_{z=1}^{Z^{T}} u_{n, m} p_{n, m, z}^{A B} \\
& R_{n}^{U}=\sum_{z=1}^{Z^{T}} v_{n, z} \times \\
&\left(\left(\sum_{m=1}^{M} u_{n, m} \min \left\{R_{n, m, z}^{U A}, R_{n, m, z}^{A B}\right\}\right)+s_{n, B} R_{n, B, z}^{U B}\right)
\end{aligned}
$$

where $\sum_{m=1}^{M} u_{n, m} \leq 1$ denotes that each GU can be assigned to one UAV which is determined by using our proposed UAV deployment scheme.

$$
\begin{array}{ll} 
& \min _{\mathbb{U}, \mathbb{S}, \mathbb{Z}} \sum_{i=1}^{N} p_{n}^{U} \\
& \text { s. t. } \\
\text { (C1) } & p_{n}^{U} \leq p_{n}^{U *} \\
\text { (C2) } & p_{m}^{A} \leq p_{m}^{A *} \\
\text { (C3) } & R_{n}^{U} \geq R_{n}^{U *} \\
\text { (C4) } & \sum_{z=1}^{Z^{T}} \sum_{m=1}^{M} u_{n, m} R_{n, m, z}^{U A} \leq \sum_{z=1} \sum_{m=1}^{M} u_{n, m} R_{n, m, z}^{A B} \\
\text { (C5) } & \sum_{m=1}^{M} u_{n, m} \gamma_{n, m, z}^{U A}+s_{n, B} \gamma_{n, B, z}^{U B} \geq \gamma_{n}^{U *} \\
\text { (C6) } & p_{n, m, z}^{U A}, p_{n, B, z}^{U B}, p_{n, m, z}^{A} \geq 0 \\
\text { (C7) } & \sum_{n \in \Omega_{S}} v_{n, z} \leq 1 \\
& Z^{T} v_{n, z}=Z_{n} \\
\text { (C8) } & \sum_{z=1} v_{m} \\
&
\end{array}
$$

In order to prolong the working life of the GUs' devices, in this paper we aim to minimize the total transmitted power of the GUs by optimizing $\mathbb{U}, \mathbb{S}$ and $\mathbb{Z}$. The optimization problem with the lowest QoS constraints can be formulated as (12). In 12, $p_{n}^{U *}$ and $p_{m}^{A *}$ are the maximum power budget of $G U_{n}$ and $U A V_{m}$ respectively. $R_{n}^{U *}$ and $\gamma_{n}^{U *}$ are minimum sum rate demand and SINR demand of the $G U_{n}$. (C4) guarantees the feasibility of the relay links. Constraint (C7) denotes that one sub-channel can be assigned to at most one direct GU, and $\Omega_{S}$ is the set of direct GUs. Constraint (C8) denotes the number of the sub-channels one GU should be occupied. Moreover, $Z_{n}$ is the minimum number of sub-channels demanded by $G U_{n}$, and it can be computed as $Z_{n}=\left\lfloor R_{n}^{\mathrm{U} *} / B_{0} \log \left(1+\gamma_{n}^{\mathrm{U} *}\right)\right\rfloor$.

\section{The Solution of The Sum Power Minimization PROBLEM}

In the power minimization problem (12), $p_{n, m, z}^{U A}, p_{n, B, z}^{U B}$, and $p_{n, m, z}^{A}$ are nonnegative and continuous variables. In addition, $u_{n, m}, s_{n, B}$ and $v_{n, z}$ are binary variables. Therefore, the transmitted power minimization problem is nonconvex and NP-hard. In order to solve it, a stepwise algorithm in our previous work [37] is adopted. In detail, the original problem is decoupled into three steps including user pairing (UP), power allocation (PA), sub-channel allocation (SA). Specifically, a location based UP scheme is proposed in this paper. The three steps are detailed as follows.

\section{A. Step 1: The location based UP scheme}

The performance of the multiple UAVs aided uplink NOMA cellular system is determined by the user pairing results. Aim to maximize the $\mathrm{SE}$ as well as the $\mathrm{EE}$ of the considered cellular uplinks, we propose a location based UP scheme. The UP scheme is to pair the direct GUs and the relay GUs. In general, a relay GU should be paired to a direct GU. Aiming to reduce the transmitted power of the relay GUs, according to 
(6), the interference introduced by their pairs should be as low as possible. Obviously, the interference introduced by direct GUs are related to their GU-UAV channels and transmitted power. Accordingly, a variable $A^{d}$ is defined to evaluate the interference level of the direct GUs and a variable $A^{r}$ is defined to evaluate the channel condition of relay GUs. Under Assumption 1, they can be calculated as

$$
\begin{gathered}
A_{j, m, z}^{d}=\left(\eta_{j, m, z}\right)^{C_{A}} \frac{\left(d_{j, B}^{U B}\right)^{\alpha_{B}}}{\left(d_{j, m}^{U A}\right)^{\alpha_{\mathrm{R}}}} \\
A_{i, m, z}^{r}=\frac{\left(\eta_{i, m, z}\right)^{C_{A}}}{\left(d_{i, m}^{U A}\right)^{\alpha_{\mathrm{R}}}\left(d_{i, B}^{U B}\right)^{\alpha_{B}}}
\end{gathered}
$$

where $C_{A}$ is a configurable hyper parameter, $\eta_{j, m, z}$ is the additional attenuation factors of the sub-channel $z$ from $G U_{j}$ to $U A V_{m}$ and $\eta_{i, m, z}$ is the additional attenuation factors of the sub-channel $z$ from $G U_{i}$ to $U A V_{m}$. In detail, $A_{j, m, z}^{d}$ is proportional to the channel gains $g_{j, m, z}^{U A}$ and the distance $d_{j, B}^{U B}$, so the larger $A_{j, m, z}^{d}$ is, $G U_{j}$ will introduce more interference to $U A V_{m}$. Likewise, $A_{i, m, z}^{r}$ is proportional to the channel gains $g_{i, m, z}^{U A}$ and is inversely proportional to the distance $d_{i, B}^{U B}$, so the smaller $A_{i, m, z}^{r}$ is, the worse $G U_{i}$ 's channel condition is.

We define a matrix $\mathbb{A}^{r}$ with a size of $1 \times N / 2$ to store the values of $A^{r}$ between the relay GUs and their assigned UAVs. In addition, a matrix $\mathbb{A}^{d}$ with a size of $M \times N / 2$ is defined to store the values of $A^{d}$ between the direct GUs and all the UAVs. Therefore, the proposed location based UP scheme is achieved by using two sorting steps as follows. Firstly, $\mathbb{A}^{r}$ is sorted in ascending order. If the relay GUs with large $A^{r}$ and direct GUs with small $A^{d}$ are paired, while the relay GUs with small $A^{r}$ and direct GUs with large $A^{d}$ are paired, the performance of the relay GUs with high channel gains and their pairs will be selfishly optimized. To be fair, the relay GUs will select their feasible partners in the above $\mathbb{A}^{r}$ order, i.e. the relay GUs with small $A^{r}$ will be paired with the direct GUs with small $A^{d}$ first. Secondly, when one relay GU connected to $U A V_{m}$ selects its partner, the $m$-th row of $\mathbb{A}^{d}$ can be sorted in ascending order and the first direct GU will be paired with the relay GU. Especially, the direct GUs that have been paired should be removed from $\mathbb{A}^{d}$ during the following user pairing for avoiding assignment conflicts. When all the GUs have been paired together, the location based UP scheme is completed.

\section{B. Step 2: Minimum SINR demands based PA}

In order to minimize the sum transmitted power of the GUs, the transmitted power of the GUs and the UAVs on each sub-channel can be initialized based on their minimum SINR demands. Under Assumption 1, the initial PA of every GU pair can be computed based on (6)-(8) as follows.

$$
p_{j, B, z}^{U B}=\frac{\gamma_{j, B, z}^{U B} N_{o} B_{o}}{g_{j, B, z}^{U B}}
$$

$$
\begin{aligned}
p_{i, m, z}^{U A}= & \frac{\gamma_{i, m, z}^{U A} N_{o} B_{o} g_{i, m, z}^{A B}\left(g_{j, B, z}^{U B}+g_{j, m, z}^{U A} \gamma_{j, B, z}^{U B}\right)}{\tau \gamma_{i, m, z}^{U A} g_{a, b, z} g_{i, B, z}^{U B} g_{j, B, z}^{U B}+g_{i, m, z}^{U A} g_{i, m, z}^{A B} g_{j, B, z}^{U B}}+ \\
& \frac{\tau \gamma_{i, m, z}^{U A} \gamma_{i, m, z}^{A B} N_{o} B_{o} g_{a, b, z} g_{j, B, z}^{U B}\left(1+\gamma_{j, B, z}^{U B}\right)}{\tau \gamma_{i, m, z}^{U A} g_{a, b, z} g_{i, B, z}^{U B} g_{j, B, z}^{U B}+g_{i, m, z}^{U A} g_{i, m, z}^{A B} g_{j, B, z}^{U B}}
\end{aligned}
$$

$$
\begin{aligned}
p_{i, m, z}^{A B}= & \frac{\gamma_{i, m, z}^{A B} N_{o} B_{o} g_{i, m, z}^{U A} g_{j, B, z}^{U B}\left(1+\gamma_{j, B, z}^{U B}\right)}{\tau \gamma_{i, m, z}^{U A} g_{a, b, z} g_{i, B, z}^{U B} g_{j, B, z}^{U B}+g_{i, m, z}^{U A} g_{i, m, z}^{A B} g_{j, B, z}^{U B}}- \\
& \frac{\gamma_{i, m, z}^{U A} N_{o} B_{o} g_{i, B, z}^{U B}\left(g_{j, B, z}^{U B}+\gamma_{j,, z}^{U B} g_{j, m, z}^{U A}\right)}{\tau \gamma_{i, m, z}^{U A} g_{a, b, z} g_{i, B, z}^{U B} g_{j, B, z}^{U B}+g_{i, m, z}^{U A} g_{i, m, z}^{A B} g_{j, B, z}^{U B}}
\end{aligned}
$$

Based on our proposed UP scheme, the channel gains between UAV and relay GUs is higher than channel gains between UAV and direct GUs, while the channel gains between BS and relay GUs is lower than channel gains between BS and direct GUs, i.e. $g_{i, m, z}^{U A} \geq g_{j, m, z}^{U A}, g_{i, B, z}^{U B} \leq g_{j, B, z}^{U B}$. Therefore (17) is always larger than 0 which satisfies the constraint (C6).

\section{Step 3: MP algorithm based SA}

According to the channel model of the relay channels in (2) and (3), it can be found that the channel gains of the relay channels have no relations to the frequency bands of the subchannels. Therefore, according to (6) and (7), the sum rates of the relayed GUs are only determined by the interference from the direct GUs on the same sub-channels and the RSI of the UAVs. As a result, it is reasonable to just focus on the SA for the direct GUs. Accordingly, the original resource allocation problem can be simplified as a SA problem for the direct GUs. To solve this problem, a stepwise scheme is adopted.

1) Sub-step 1: We temporarily ignore the power constraints, (12) can be reformulated as (18) based on the initial PA results.

$$
\begin{array}{ll} 
& \min _{\mathbb{Z}} \sum_{n \in \Omega_{S}} \sum_{z=1}^{Z^{T}} v_{n, z} p_{n, z}^{\prime} \\
& \text { s. t. } \\
\left(C 1^{\prime}\right) & v_{n, z} \in\{0,1\} \\
\left(C 2^{\prime}\right) & \sum_{n \in \Omega_{S}} v_{n, z} \leq 1 \\
\left(C 3^{\prime}\right) & \sum_{z=1}^{Z^{T}} v_{n, z}=Z_{n}
\end{array}
$$

where $\Omega_{S}$ is the set of direct GUs and $p_{n, z}^{\prime}$ is the total transmitted power of the $n$-th GU pair on sub-channel $z$. According to Assumption 1, it is easy to achieve that $p_{j, z}^{\prime}=$ $p_{j, B, z}^{U B}+p_{i, m, z}^{U A}$. Here problem $\sqrt{18}$ is solved by the message passing (MP) algorithm [37]- 39 with iterative computations, the details can be found in Appendix A. Under Assumption $\mathbf{1}$, the SA results of the relayed GUs are determined by direct GUs as follows

$$
v_{i, z}=v_{j, z} .
$$

2) Sub-step 2: After the step of SA, the total power consumption of the GUs and the UAVs can be computed according to $(9)$ and $(10)$. Since the power constraints are 
removed in sub-step 1, the SA results for the GUs should be rechecked according to (C1) and (C2). Based on SIC, the interference to the direct GUs can be totally canceled at the BS, their power constraints can be independently checked without considering the access status of the relay GUs. However, the transmitted power of the relay GUs is related to the access status of the direct GUs. Besides, the transmitted power of the UAVs is dependent on the access status of both the relay GUs and the direct GUs. Therefore, it is necessary to conduct access control (AC) for the direct GUs, the relay GUs and the UAVs in sequences. Under Assumption 1, a soft AC scheme [37] can be considered as follows.

Firstly, if the total power of $G U_{j}$ is larger than its power budget, the highest power $p_{j, B, z}^{U B}$ will be reduced and the $G U_{j}$ is refused to access the corresponding sub-channel $z$. Then, its total power can be recomputed and rechecked again. If $p_{j}^{U} \leq p_{j}^{U *}$, the soft access control scheme for $G U_{j}$ is finished and the present PA and SA results are regarded as the optimal results for $G U_{j}$. Otherwise, the soft access control scheme will be carried out continuously, until the power budget constraint can be satisfied.

Secondly, according to the AC results for the direct GUs, the initialized power of the relayed GUs should be rechecked. In detail, if the power of $G U_{j}$ on sub-channel $z$ is reduced, $G U_{i}$ won't be interfered by $G U_{j}$ and the minimum required power for $G U_{i}$ on sub-channel $z$ can be recomputed as

$$
p_{i, m, z}^{U A}=\frac{\gamma_{i, m, z}^{U A}\left(N_{o} B_{o}+\tau g_{a, b, z} p_{i, m, z}^{A B}\right)}{g_{i . m . z}^{U A}}
$$

According to the updated power, the total power of $G U_{i}$ can be achieved according to (9). Then the total power should be rechecked by the soft $\mathrm{AC}$ scheme similar to that for the direct GUs.

Thirdly, According to the AC results for the direct GUs and the relay GUs, the power of the UAVs on each subchannel should also be updated based on (21) where $I_{e}=$ $\tau \gamma_{i, m, z}^{U A} g_{a, b, z} g_{i, B, z}^{U B}, I_{g}=\gamma_{i, m, z}^{U A} g_{i, B, z}^{U B}\left(g_{j, B, z}^{U B}+\gamma_{j, B, z}^{U B} g_{j, m, z}^{U A}\right)$. Based on the updated power results of the UAVs, the total transmitted power of the UAVs can be computed as in 10 . Then the power budget constraint (C2) can also be rechecked for the UAVs by the soft AC scheme,

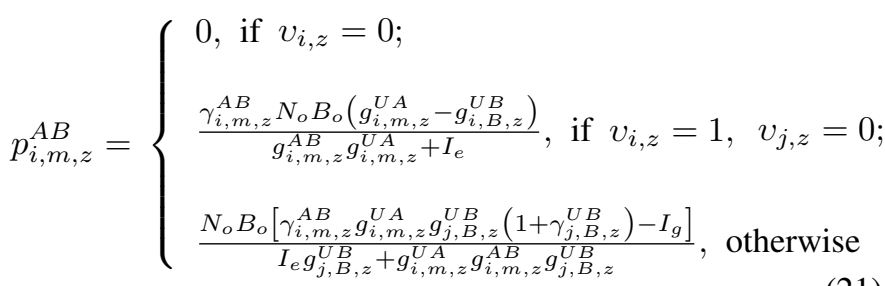

Additionally, since the UAVs are utilized as the relays for the relay GUs, their AC results influence the access status of the relay GUs. In detail, if $p_{i, m, z}^{A B}$ is reduced, then $u_{i, m}=0$, $v_{i, z}=0$ and $p_{i, m, z}^{U A}=0$, which means $G U_{i}$ is refused to use sub-channel $z$. After the UP, PA, SA steps, the EE and SE of the uplinks can be further optimized with the optimal PA and $\mathrm{SA}$ results based on multiple UAVs aided NOMA.

\section{Simulation Results And AnAlysis}

This section evaluates the SE and EE performances of the proposed multiple UAVs aided uplink NOMA through extensive simulations. In detail, we simulate four uplink NOMA schemes, including ground to ground (G2G) uplink NOMA without UAVs, single UAV aided uplink NOMA (single UAV), multiple UAVs aided uplink NOMA with random UAV deployment (Multi-UAV-r) and the proposed Multi-UAV-K-cluster scheme for comparisons.

In single UAV based cellular NOMA scheme, the UAV is placed above the BS with an initial height. All the GUs will compute their own $A^{d}$ based on 13 and all $A^{d}$ will be sorted in descending order. The first half GUs have higher $A^{d}$, thus they will be assigned to communicate with UAVs. In addition, the left GUs will be assigned to communicate with BS directly. For fairness, the first half of GUs will be paired with the last half of GUs with the sorted order for the $A^{d}$.

In multi-UAV-r scheme, $M$ UAVs are randomly and uniformly deployed in the cell with initial heights. Same to the multi-UAV-K-cluster scheme, the heights of the UAVs will be adjusted until the UAV-BS channels are LoS. The receiver determination and user pairing are detailed as follows. We define a matrix $\mathbb{A}_{r}^{d}$ with size of $M \times N$ to store the $A^{d}$ values between $M$ UAVs and $N$ GUs. Firstly, every column of $\mathbb{A}_{r}^{d}$ is sort in descending order and the UAV with largest $A^{d}$ value is the best UAV for the GU to select. The number of every GU's best UAV is recorded in vector $\mathbb{I}_{1 \times N}$. Secondly, the largest $A^{d}$ of every GU are sorted in descending order and the results are recorded in vector $\mathbb{K}_{1 \times N}$. Then the first half of GUs in $\mathbb{K}_{1 \times N}$ are assigned to communicate with UAVs and the other GUs communicate with the BS directly. After receiver determination, the user pairing steps are same to multi-UAVK-cluster scheme.

For simulations, a ground cellular network is considered with a radius of 500 meters. In this cellular network, 32 GUs require uplink transmissions at the same time. In detail, these GUs are randomly and uniformly distributed within the coverage of the cellular network. Moreover, for the MultiUAV-r scheme and the Multi-UAV-K-cluster scheme, the numbers of the UAVs are both set as $M=4$. The initial height of the UAVs is assumed as 200 meters. The distance between antenna $a$ and antenna $b$ is set as $d_{a b}=1 \mathrm{~m}$. The residual self-interference coefficient is set as $\tau=-100 \mathrm{~dB}$. The parameter values for the simulated scenario are concluded in Table. III

Followed by the scenario considerations, the channel parameters are set as follows. At first, the total number of the available sub-channels is 32. Then the unit bandwidth of each sub-channel is $0.3125 \mathrm{MHz}$ and the power spectral density of the noise is $5 \times 10^{-20}$ watt/Hz. Moreover, for the largescale fading, the path losses of the GU-UAV and the GU-BS channels are $\alpha_{R}=2$ and $\alpha_{B}=4$ respectively. Meanwhile, the additional attenuation factors for the LoS and NLoS channels are $\eta_{L o S}=-1 \mathrm{~dB}$ and $\eta_{N L o S}=-20 \mathrm{~dB}$. Especially, we just consider an urban environment for simulating the schemes. Thus, according to [40], the LoS probability related parameters are set as $\psi=9.6$ and $\beta=0.16$ for the simulated urban 
TABLE I: Parameter setting of the simulation scenario.

\begin{tabular}{|c|c|}
\hline Parameters & Values \\
\hline The radius of the cellular network & $R=500$ meter \\
\hline Number of GU & $N=32$ \\
\hline Number of UAV & $M=4$ \\
\hline Number of sub-channel & $Z^{T}=32$ \\
\hline Unit bandwidth of each sub-channel & $B_{0}=0.3125 \mathrm{MHz}$ \\
\hline The power spectral density of the noise & $N_{0}=5 \times 10^{-20} \mathrm{Watt} / \mathrm{Hz}$ \\
\hline The path loss of GU-UAV channels & $\alpha_{R}=2$ \\
\hline The path loss of GU-BS channels & $\alpha_{B}=4$ \\
\hline $\begin{array}{l}\text { The additional attenuation factor } \\
\text { for the LoS channel }\end{array}$ & $\eta_{L o S}=-1 \mathrm{~dB}$ \\
\hline $\begin{array}{l}\text { The additional attenuation factor } \\
\text { for the NLoS channel }\end{array}$ & $\eta_{N L o S}=-20 \mathrm{~dB}$ \\
\hline The power budget of each UAV & $P_{U A V}=3$ Watt \\
\hline The power budget of each GU & $P_{G U}=0.5$ Watt \\
\hline The distance between two antennas of UAV & $d_{a b}=1$ meter \\
\hline The residual self-interference coefficient & $\tau=-100 \mathrm{~dB}$ \\
\hline
\end{tabular}

TABLE II: The definition of performance criteria.

\begin{tabular}{|c|c|}
\hline Performance Criterion & Definition \\
\hline Energy Efficiency (EE) of GUs & $\sum R^{U} / \sum P^{U}$ \\
\hline $\begin{array}{c}\text { Normalized access ratios } \\
\text { of sub-channels }\end{array}$ & $\begin{array}{c}\text { The number of sub-channels } \\
\text { used by UAVs and GUs) } / Z^{T}\end{array}$ \\
\hline
\end{tabular}

environment. Furthermore, the power budgets of each UAV and each GU are set as 3 watt and 0.5 watt. For simplicity, the simulated QoS demands of all the GUs are equally considered as $\gamma_{n}^{U *}=\gamma^{*}, R_{n}^{U *}=R^{*}$ and $Z_{n}=2, \forall n \in\{1, \cdots, N\}$. The simulation performances have been averaged by 3,000 Monte Carlo experiments and the definitions of the critical performances are given in Table. II

Firstly, Fig. 3 shows the EE performance of the four schemes in term of the varying SINR target of the GUs. Also it shows that both the Multi-UAV-r scheme and the Multi-UAV$\mathrm{K}$-cluster scheme significantly outperform the single UAV scheme and G2G scheme, especially with a low GUs' SINR target. It can be explained according two reasons. One reason is that most of the GU-UAV channels are LoS channels which demand dramatically low power for the GUs to transmit information under the same SINR targets. Another reason is that benefitting from the high channel gains of the GU-UAV channels, the BS is able to remove the interference from the direct GUs perfectly with SIC, which increases the normalized access ratio for the sub-channels.

Apart from the conclusions above, from Fig. 3 it can also be observed that the Multi-UAV-K-cluster scheme always outperforms Multi-UAV-r scheme. It can be explained that, for the Multi-UAV-K-cluster based UAV deployment scheme, the UAVs are placed on more accurate locations for relay GUs than under the random UAV deployment. Accordingly, the relay GUs will have much higher channel gains to the UAVs based on K-means clustering.

Moreover, with the increasing of the GUs' SINR target, the EE performances of all the schemes degrade significantly. It is reasonable since the sum power of GUs increases to satisfy

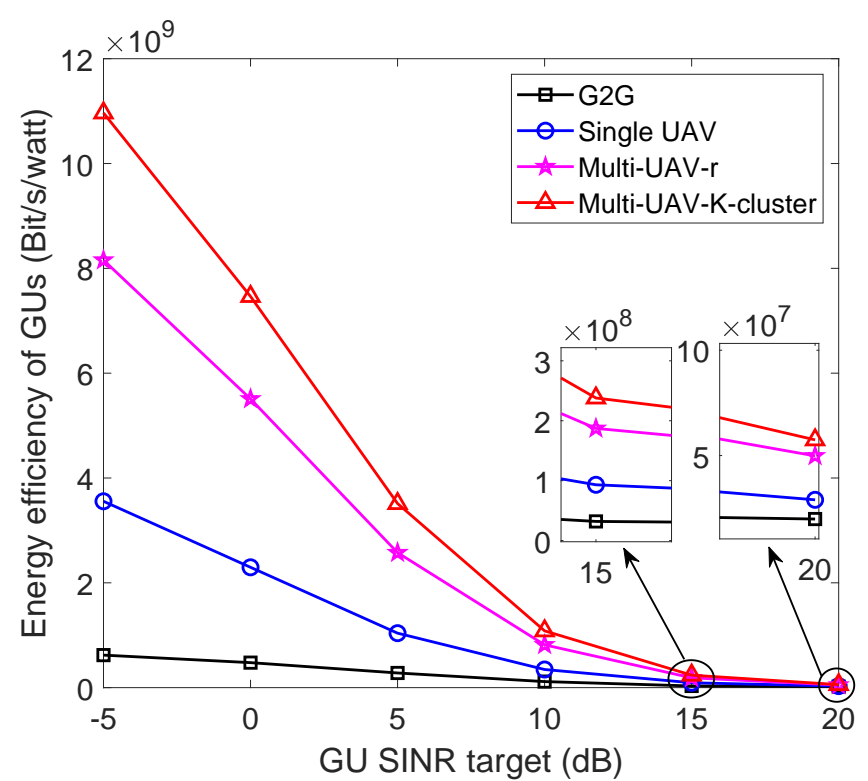

Fig. 3: The EE performance of GUs in term of GUs' SINR target.

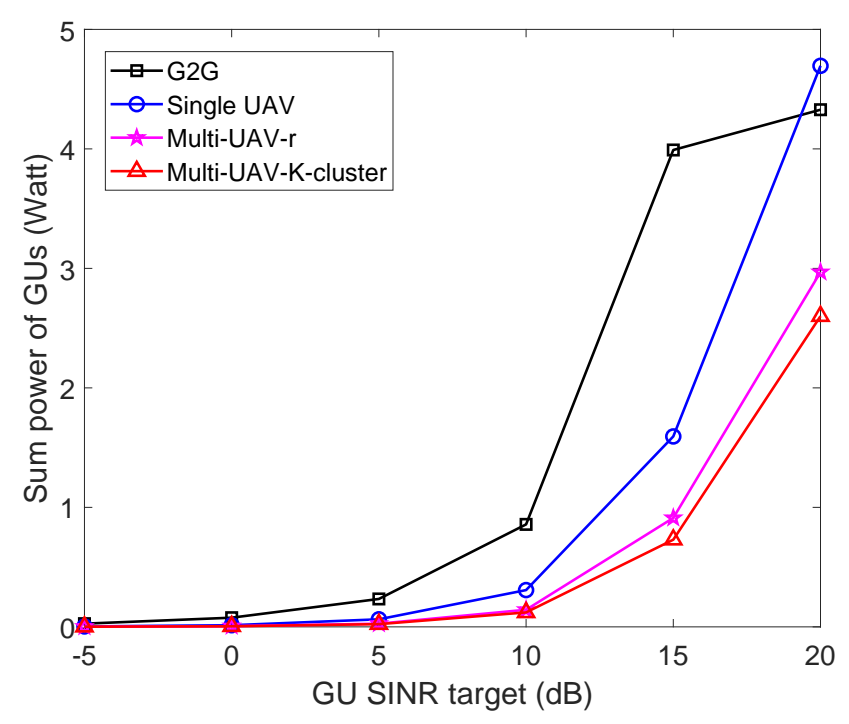

Fig. 4: The sum power of the GUs in term of GUs' SINR target.

the QoS of the GUs. Then, it definitely introduces higher interference to the relay GUs and causes decrease drop of the normalized access ratios for the sub-channels. Fig. 4 shows the sum transmitted power of GUs in term of GUs' SINR target. From Fig. 4 we can see that the transmitted power of GUs increases with the GUs' SINR target increases. And under Multi-UAV-K-cluster scheme, GUs demand lowest transmitted power. Especially, the sum power of the GUs decreases in the G2G scheme when the GUs' SINR target exceeds $15 \mathrm{~d}$ B. It is due to the reason that the co-channel interference is too high to enable the paired GUs to share the sub-channels with the G2G scheme. Fig. 5 shows the normalized access ratios of sub-channels in term of GUs' SINR target. It shows that 


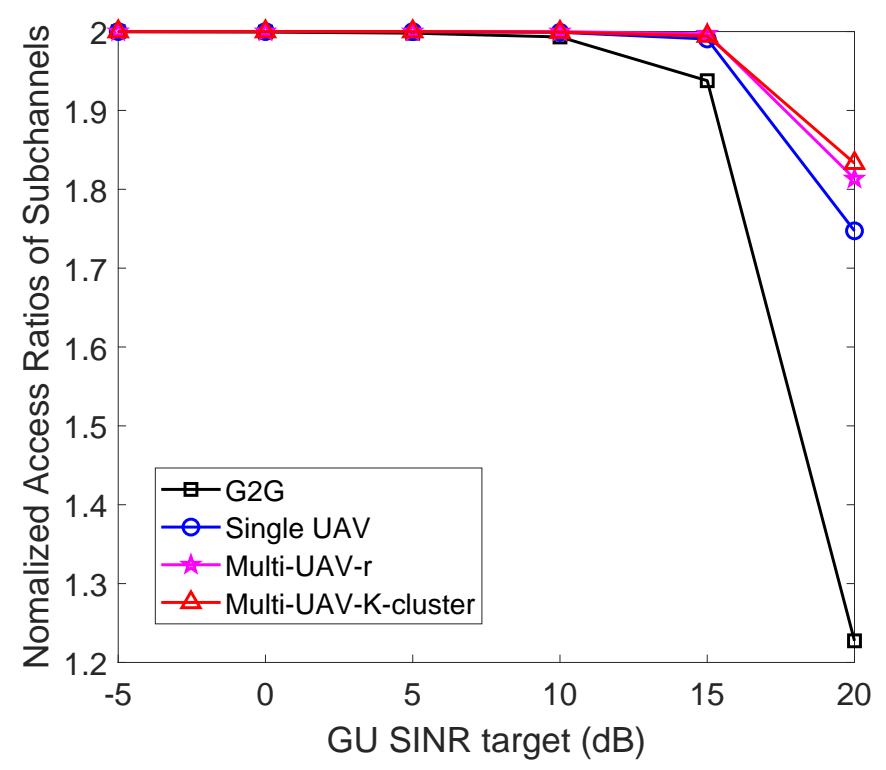

Fig. 5: Nomalized access ratios of subchannels in term of GUs' SINR target.

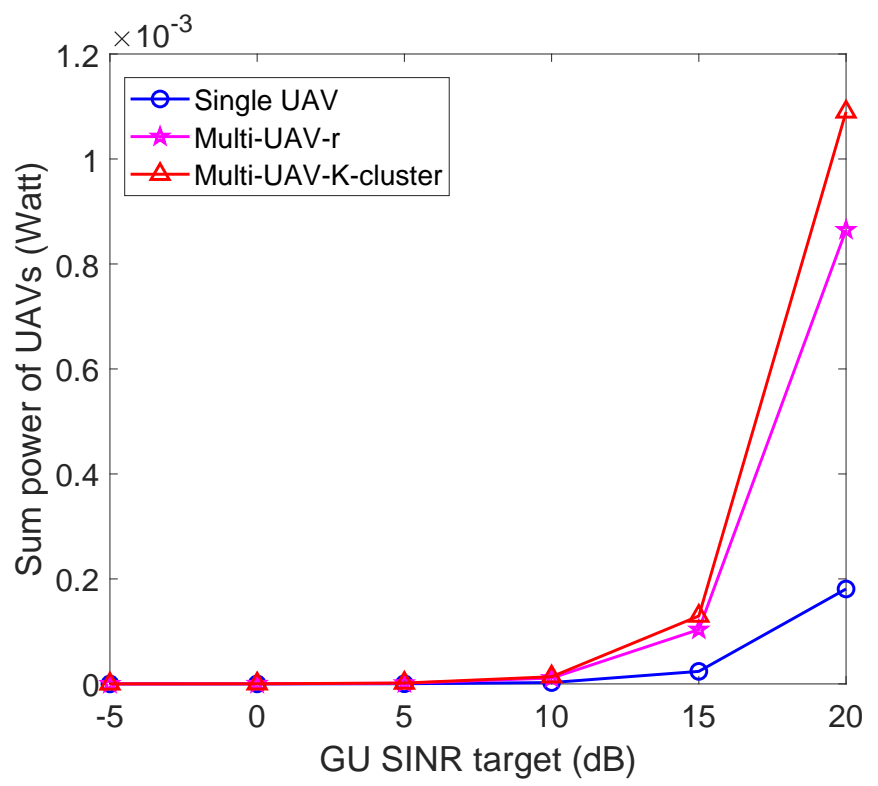

Fig. 6: The sum power of UAVs in term of GUs' SINR target.

when the GUs' SINR target exceeds $15 \mathrm{~dB}$, the normalized access ratios of sub-channels under $\mathrm{G} 2 \mathrm{G}$ scheme decrease dramatically, while that under Multi-UAV-K-cluster scheme still keep high level.

Furthermore, in order to evaluate the feasibility of the proposed multiple UAVs aided uplink NOMA, the sum power of the UAVs has been simulated and the corresponding result is depicted in Fig. 6. Compared with the results in Fig. 4, it can be found that the sum power of the UAVs is much less than that of the GUs. As a result, the proposed schemes can achieve higher EE and SE for the uplinks with a limited power cost of the UAVs.

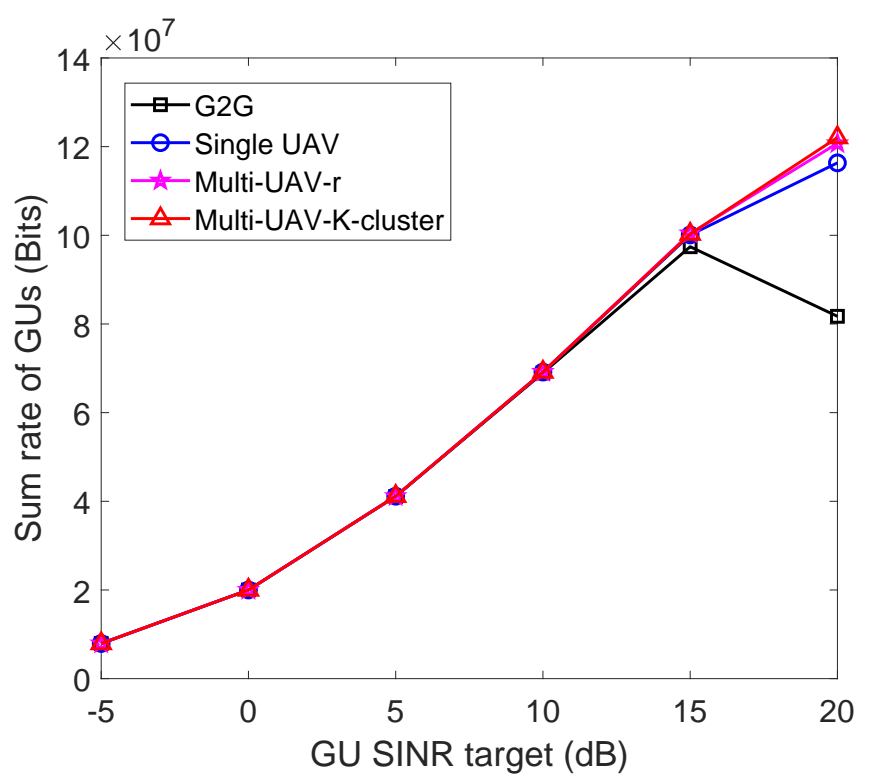

Fig. 7: The sum rate of the GUs in term of GUs' SINR target.

TABLE III: The number of UAVs used in multi-UAV-K-cluster scheme under $M=4$.

\begin{tabular}{|c||c||c|}
\hline The number of UAVs actually used & Count & Percent $(\%)$ \\
\hline 3 & 245 & 8.2 \\
\hline 4 & 2755 & 91.8 \\
\hline
\end{tabular}

In Fig. 7, the sum rates of the GUs are simulated in term of the GUs' SINR target. Since we consider a sum power minimization based resource allocation strategy with the lowest QoS constraints, the sum rates of the GUs in the four schemes are same under low GUs' SINR targets (i.e. -5 $\mathrm{dB}$ and $-10 \mathrm{~dB})$. When the GUs' SINR targets are larger than $15 \mathrm{~dB}$, the sum rate of the proposed multi-UAV-K-cluster scheme still increases with the increase of the GUs' SINR targets. However, the sum rate of the GUs in the single UAV scheme decrease slightly and that of G2G scheme decrease significantly. This is due to the fact that under high GUs' SINR target, high channel gains for the GU-UAV channels and UAV-BS channels can still resist the interference introduced by direct GUs successfully without exceeding the power budgets of GUs.

Besides the outstanding performance in EE and SE, another advantage of the proposed multi-UAV-K-cluster scheme is that when the GUs' locations are relatively concentrated, the actual number of the clusters might be less than the number of the UAVs. In this case, less UAVs can be occupied to serve the GUs with no loss of the system SE and EE. When the number of UAVs is set as $M=4$, among 3,000 Monte Carlo experiments, there are 245 times of the simulations accomplished the transmission by using 3 UAVs, see Table. III. 


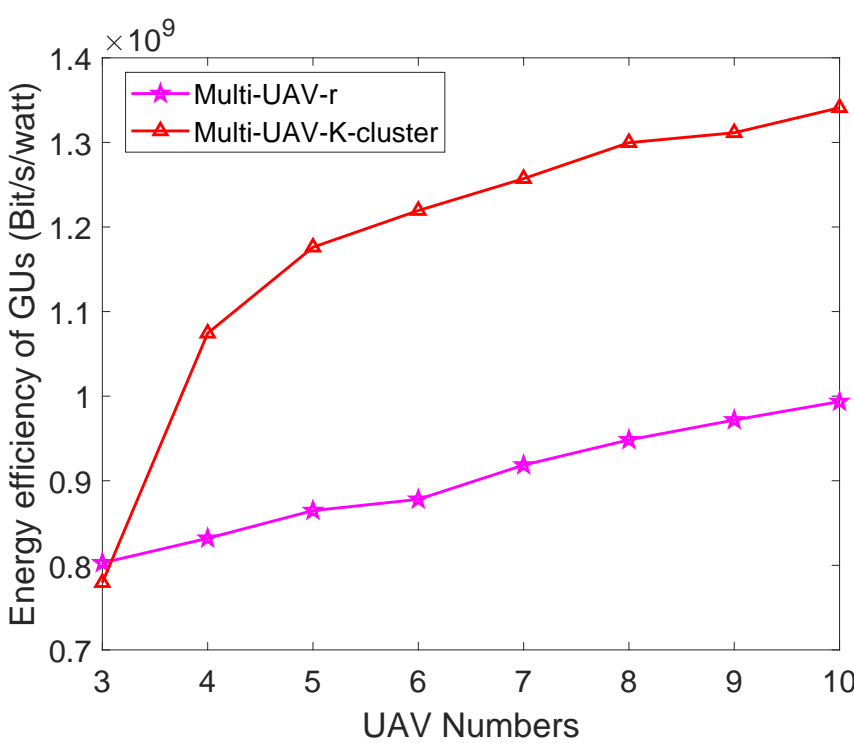

Fig. 8: The EE performance of GUs in term of the number of UAVs when the GUs' SINR target is $10 \mathrm{~dB}$.

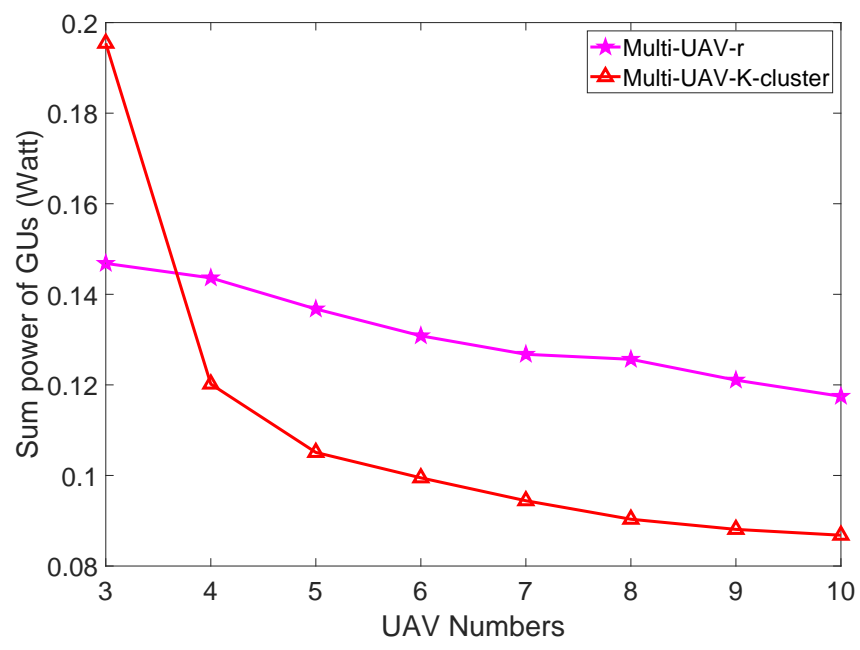

Fig. 9: The sum power of GUs in term of the number of the UAVs when the GUs' SINR target is $10 \mathrm{~dB}$.

After comparing the $\mathrm{EE}$ and the SE performances of the uplinks with the four schemes, we investigate multiple performances of the proposed multiple UAVs aided uplink NOMA technology in term of the UAVs' number. For saving space, we just investigate them when the GUs' SINR target is $10 \mathrm{~dB}$. On the EE performance, Fig. 8 shows its changing tendency with the growth of the UAVs' number. Fig. 8 demonstrates that the uplink EEs for both the Multi-UAV-r scheme and the multi-UAV-K- cluster scheme continuously get higher when the UAVs' number increases. It is reasonable that the more the UAVs are, the better the GU-UAV channels can be. With the GU-UAV channels become better, the transmitted power of relay GUs will be lower under the same GUs' SINR targets. As a result, the interference introduced to direct GUs by relay GUs will be smaller under above case, which will make direct GUs consume less transmitted power and will enhance the EE of the system. Therefore, the sum power of the GUs is also decreasing with the number of the UAVs increases. Fig. 9 shows the sum transmitted power of GUs in term of the number of UAVs. From Fig. 9 we can obtain that the sum transmitted power of GUs decreases with the number of UAVs increases.

Fig. 8 and Fig. 9 demonstrate that the Multi-UAV-Kcluster scheme significantly outperforms the Multi-UAV-r scheme because of its accurate UAV deployment by using the GUs' location information. Besides, the Multi-UAV-K-cluster scheme achieves the outperformance with the optimized UAV number. Table. III shows the number of actually used UAVs when $M=4$. To be more concise, the average number of the actually used UAVs from the 3,000 Monte Carlo experiments for two multiple UAVs schemes in term of the number of UAVs are depcited in Fig. 10. From this figure, we can obtain that the Multi-UAV-K-cluster scheme can achieve better performances with less UAVs. When the number of the available UAVs is more than 6 , the multiUAV-K-cluster scheme uses one less UAVs than multi-UAV-r scheme statistically.

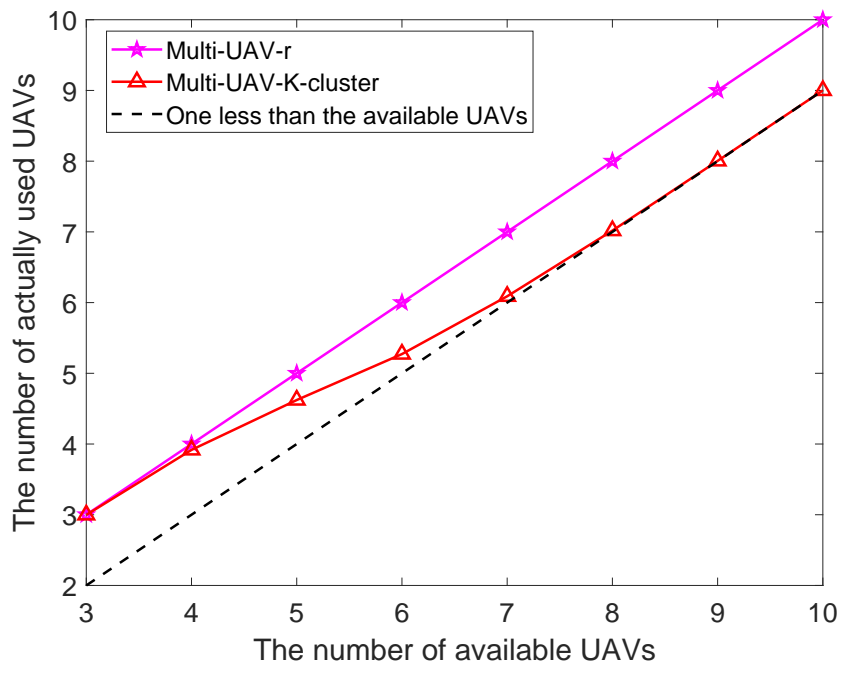

Fig. 10: The average number of the actually used UAVs in term of the number of available UAVS.

\section{CONCLUSION}

This paper proposed a multiple UAVs aided uplink NOMA technology to improve the energy efficiency and spectral efficiency of the GUs' uplink transmission in cellular networks. Moreover, a multi-UAV-K-cluster based UAV deployment scheme and a location based user pairing scheme have been developed to optimize the performances of the multiple UAVs aided uplink NOMA. Furthermore, a sum power minimization based resource allocation scheme was formulated to evaluate the performance of the optimized uplink transmissions based on the proposed schemes. At last, exhaustive experiments were conducted for comparing the proposed multiple UAVs aided NOMA scheme with the single UAV aided NOMA scheme and traditional NOMA with no helps from the UAVs. Simulation results confirmed that the 
proposed multiple UAVs aided NOMA significantly improves the energy efficiency and spectral efficiency of the GUs' uplinks with the optimized deployment, pairing and resource allocation schemes.

\section{APPENDIX A}

In our problem, the iterative message passing process is as follows

$$
\begin{aligned}
& \mu_{n, z}^{(t+1)}=p_{n, z}^{\prime}-\rho_{n}\left\{\mu_{f, n}^{(t)}+p_{n, z}^{\prime}\right\}_{Z_{n}^{\text {th }}} \\
& -\left(1-\rho_{n}\right)\left(p_{n, z}^{\prime}+\mu_{z, n}^{(t)}\right), f \neq z \\
& \mu_{z, n}^{(t+1)}=-\rho_{n} \min _{l \neq n} \mu_{l, z}^{(t+1)}-\left(1-\rho_{n}\right) \mu_{n, z}^{(t+1)} \\
& \tau_{n, z}^{(t)}=\mu_{z, n}^{(t)}+\mu_{n, z}^{(t)} \\
& v_{n, z}= \begin{cases}1 & \text { if } \tau_{n, z}^{(t)}<0 \\
0 & \text { otherwise }\end{cases} \\
& v_{n, z}^{T}=v_{n, z}^{T-1}, \forall n, z
\end{aligned}
$$

The process from (22) to (25) will iterate continuously until (26) is satisfied at the $T$ time, when the optimal SA result is obtained for the $G U_{n}$. In detail, $\rho_{n}$ is a weight for formulating the messages and $\left\{\mu_{f, n}^{(t)}+p_{n, f}^{\prime}\right\}_{Z_{n}^{\text {th }}}$ denotes the $Z_{n}$-th value of $\mu_{f, n}^{(t)}+p_{n, f}^{\prime}$ when it is sorted in ascending order with the constraint condition of $f \neq z$. In general, the message $\mu_{n, z}$ from $G U_{n}$ to sub-channel $z$ and the message $\mu_{z, n}$ from subchannel $z$ to $G U_{n}$ are initialized by $\mu_{n, z}^{0}=0$ and $\mu_{z, n}^{0}=0$, respectively.

\section{REFERENCES}

[1] R. W. Heath, "Going toward 6G," IEEE Signal Process. Mag., vol. 36, no. 3, pp. 3-4, 2019.

[2] Y. Cai, Z. Qin, F. Cui, G. Y. Li, and J. A. Mccann, "Modulation and multiple access for 5G networks," IEEE Commun. Surv. Tutorials, vol. 20, no. 1, pp. 629-646, 2018.

[3] G. A. Akpakwu, B. J. Silva, G. P. Hancke, and A. M. Abu-Mahfouz, "A survey on $5 \mathrm{G}$ networks for the internet of things: communication technologies and challenges," IEEE Access, vol. 6, pp. 3619-3647, 2018.

[4] Z. Zhou, J. Feng, Z. Chang, and X. Shen, "Energy-efficient edge computing service provisioning for vehicular networks: a consensus ADMM approach," IEEE Trans. Veh. Technol., vol. 68, no. 5, pp. 50875099, Mar. 2019.

[5] X. Cheng, F. Lyu, W. Quan, C. Zhou, H. He, W. Shi, and X. Shen, "Space/aerial-assisted computing offloading for IoT applications: a learning-based approach," IEEE J. Sel. Areas Commun., vol. 37, no. 5, pp. 1117-1129, 2019.

[6] W. Wu, N. Zhang, N. Cheng, Y. Tang, K. Aldubaikhy, and X Shen, "Beef up mmWave dense cellular networks with D2D-assisted cooperative edge caching," IEEE Trans. Veh. Technol., vol. 68, no. 4, pp. 3890-3904, 2019.

[7] Z. Zhou, P. Liu, J. Feng, Y. Zhang, S. Mumtaz, and J. Rodriguez, "Computation resource allocation and task assignment optimization in vehicular fog computing: a contract-matching approach," IEEE Trans. Veh. Technol., vol. 68, no. 4, pp. 3113-3125, Apr. 2019.

[8] L. Dai, B. Wang, Z. Ding, Z. Wang, S. Chen, and L. Hanzo, "A survey of non-orthogonal multiple access for 5G," IEEE Commun. Surv. Tutorials, vol. 20, no. 3, pp. 2294-2323, 2018.

[9] M. Shirvanimoghaddam, M. Dohler, and S. J. Johnson, "Massive nonorthogonal multiple access for cellular IoT: potentials and limitations," IEEE Commun. Mag., vol. 55, no. 9, pp. 55-61, 2017.

[10] Q. Wu, W. Chen, D. W. K. Ng, and R. Schober, "Spectral and energy efficient wireless powered IoT networks: NOMA or TDMA?" IEEE Trans. Veh. Technol., vol. 67, no. 7, pp. 6663-6667, 2018.
[11] Z. Ding, X. Lei, G. K. Karagiannidis, R. Schober, J. Yuan, and V. Bhargava, "A survey on non-orthogonal multiple access for $5 \mathrm{G}$ networks: research challenges and future trends," IEEE J. Sel. Areas Commun., vol. 35, no. 10, pp. 2181-2195, 2017.

[12] Z. Ding, et al., "Application of non-orthogonal multiple access in LTE and 5G networks," IEEE Commun. Mag., vol. 55, no. 2, pp. 185-191, 2017.

[13] Y. Saito, Y. Kishiyama, A. Benjebbour, T. Nakamura, A. Li, and K. Higuchi, "Non-orthogonal multiple access (NOMA) for cellular future radio access," in IEEE Vehicular Technology Conference, 2013, pp. 1-4.

[14] Z. Ding, F. Adachi, and H. V. Poor, "The application of MIMO to nonorthogonal multiple access," IEEE Trans. Wirel. Commun., vol. 15, no. 1 , pp. 537-552, 2016.

[15] Z. Ding, R. Schober, and H. V. Poor, "A general MIMO framework for NOMA downlink and uplink transmission based on signal alignment," IEEE Trans. Wirel. Commun., vol. 15, no. 6, pp. 4438-4454, 2016.

[16] Z. Yang, Z. Ding, P. Fan, and N. Al-Dhahir, "A general power allocation scheme to guarantee quality of service in downlink and uplink NOMA systems," IEEE Trans. Wirel. Commun., vol. 15, no. 11, pp. 7244-7257, 2016.

[17] J. Cui, Z. Ding, and P. Fan, "A novel power allocation scheme under outage constraints in NOMA systems," IEEE Signal Process. Lett., vol. 23, no. 9, pp. 1226-1230, 2016.

[18] M. Liu, T. Song, and G. Gui, "Deep cognitive perspective: resource allocation for NOMA based heterogeneous IoT with imperfect SIC," IEEE Internet Things J., vol. 6, no. 2, pp. 2885-2894, 2019.

[19] Y. Yin, Y. Peng, M. Liu, J. Yang, and G. Gui, "Dynamic user groupingbased NOMA over Rayleigh fading channels," IEEE Access, vol. 7, pp. 110964-110971, 2019.

[20] G. Gui, H. Sari, H. Sari, and E. Biglieri, "A new definition of fairness for non-orthogonal multiple access," IEEE Commun. Lett., vol. 23, no. 7, pp. 1267-1271, 2019.

[21] M. Liu, J. Yang, and G. Gui, "DSF-NOMA: UAV-assisted emergency communication technology in a heterogeneous internet of things," IEEE Internet Things J., vol. 6, no. 3, pp. 5508-5519, 2019.

[22] Y. Liu, Z. Qin, M. Elkashlan, A. Nallanathan, and J. A. Mccann, "Nonorthogonal multiple access in large-scale heterogeneous networks," IEEE J. Sel. Areas Commun., vol. 35, no. 12, pp. 2667-2680, 2017.

[23] X. Zhu, C. Jiang, L. Kuang, N. Ge, and J. Lu, "Non-orthogonal multiple access based integrated terrestrial-satellite networks," IEEE J. Sel. Areas Commun., vol. 35, no. 10, pp. 2253-2267, 2017.

[24] Y. Liu, Z. Qin, Y. Cai, Y. Gao, G. Li, and A. Nallanathan, "UAV communications based on Non-orthogonal multiple access," IEEE Wireless Commun., vol. 26, no. 1, pp. 52-57, 2019.

[25] N. Zhao et al., "Joint trajectory and precoding optimization for UAVassisted NOMA networks," IEEE Trans. Commun., vol. 67, no. 5, pp. 3723-3735, 2019.

[26] J.-B. Seo, S. Pack, and H. Jin, "Uplink NOMA random access for UAVassisted communications," IEEE Trans. Veh. Technol., vol. 68, no. 8, pp. 8289-8293, 2019.

[27] R. Duan, J. Wang, C. Jiang, H. Yao, Y. Ren, and Y. Qian, "Resource allocation for multi-UAV aided IoT NOMA uplink transmission systems," IEEE Internet Things J., vol. 6, no. 4, pp. 7025-7037, 2019.

[28] H. Menouar, I. Guvenc, K. Akkaya, A. S. Uluagac, A. Kadri, and A. Tuncer, "UAV-enabled intelligent transportation systems for the smart City: applications and challenges," IEEE Commun. Mag., vol. 55, no. 3, pp. 22-28, 2017.

[29] Y. Zeng, R. Zhang, and T. J. Lim, "Wireless communications with unmanned aerial vehicles: opportunities and challenges," IEEE Commun Mag., vol. 54, no. 5, pp. 36-42, 2016.

[30] F. Tang, Z. Md. Fadlullah, N. Kato, F. Ono, and R. Miura, "AC-POCA: anti-coordination game based partially overlapping channels assignment in combined UAV and D2D based networks," IEEE Trans. Veh. Technol., vol. 67 , no. 2, pp. 1672-1683, 2018.

[31] N. Zhang, S. Zhang, P. Yang, O. Alhussein, W. Zhuang, and X. Shen, "Software defined space-air-ground integrated vehicular networks: challenges and solutions," IEEE Commun. Mag., vol. 55, no. 7, pp. 101109,2017

[32] N. Kato et al., "Optimizing space-air-ground integrated networks by artificial intelligence," IEEE Wirel. Commun., vol. 26, no. 4, pp. 140147, 2019.

[33] F. Cui, Y. Cai, Z. Qin, M. Zhao, and G. Y. Li, "Multiple access for mobile-UAV enabled networks: joint trajectory design and resource allocation," IEEE Trans. Commun., vol. 67, no. 7, pp. 4980-4994, 2019.

[34] M. T. Nguyen and L. B. Le, "NOMA user pairing and UAV placement in UAV-based wireless networks," in International Conference on Communications (ICC), 2019, pp. 1-6. 
[35] V.-L. Dao, H. Tran, S. Girs, and E. Uhlemann, "Reliability and fairness for UAV communication based on non-orthogonal multiple access," in IEEE International Conference on Communications Workshops (ICC Workshops), 2019, pp. 1-6.

[36] Z. Xue, J. Wang, G. Ding, Q. Wu, Y. Lin, and T. A. Tsiftsis, "Device-toIEEE Access, vol. 6, pp. 34488-34502, 2018.

[37] M. Liu, G. Gui, N. Zhao, J. Sun, H. Gacanin, and H. Sari, "UAVaided air-to-ground cooperative non-orthogonal multiple access," IEEE Internet Things J., to be published, doi: 10.1109/JIOT.2019.2957225.

[38] M. Liu, T. Song, J. Hu, J. Yang, and G. Gui, "Deep learning-inspired message passing algorithm for efficient resource allocation in cognitive radio networks," IEEE Trans. Veh. Technol., vol. 68, no. 1, pp. 641-653, 2019.

[39] A. Abrardo, M. Belleschi, P. Detti, and M. Moretti, "Message passing resource allocation for the uplink of multi-carrier multi-format systems," IEEE Trans. Wirel. Commun., vol. 11, no. 1, pp. 130-141, 2012.

[40] M. F. Sohail, C. Y. Leow, and S. Won, "Non-orthogonal multiple access for unmanned aerial vehicle assisted communication," IEEE Access, vol. 6, pp. 22716-22727, 2018.

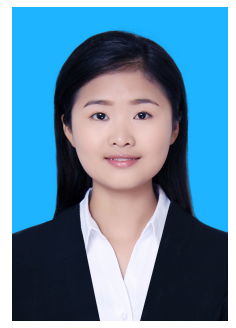

Jie Wang (S'18) received the B.S. degree from the College of Automation \& College of Artificial Intelligence, Nanjing University of Posts and Telecommunications (NJUPT), Nanjing, China, in 2015, where she is currently pursuing the Ph.D. degree. Her research interests include deep learning, channel estimation, and resource allocation and its application in wireless communications device communications underlying UAV-supported social networking,"

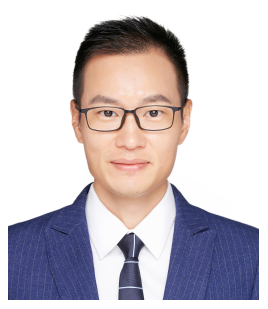

Guan Gui (M'11-SM'17) is currently a professor with Nanjing University of Posts and Telecommunications, Nanjing, China. He received the Dr. Eng degree in Information and Communication Engineering from University of Electronic Science and Technology of China, Chengdu, China, in 2012. Dr. Gui has published more than 200 international peerreviewed journal/conference papers. He received Member and Global Activities Contributions Award in IEEE ComSoc and 9 best paper awards including ICC 2017, ICC 2014 and VTC 2014-Spring. He was also selected as for Jiangsu Specially-Appointed Professor (2016), Jiangsu High-level Innovation and Entrepreneurial Talent (2016), Jiangsu Six Top Talent (2018), Nanjing Youth Award (2018). He is serving or served on the editorial boards including IEEE Transactions on Vehicular Technology, Physical Communication, Wireless Networks,IEEE Access, since 2018. He is IEEE Senior Member.

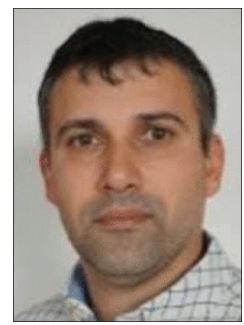

Haris Gacanin [SM] (haris.gacanin@ nokia-belllabs.com) received his Dipl.-Ing. degree in electrical engineering from the University of Sarajevo in 2000. In 2005 and 2008, respectively, he received M.Sc. and Ph.D. degrees from Tohoku University, Japan. He was with Tohoku University from 2008 until 2010, first as a Japan Society for Promotion of Science postdoctoral fellow and later as an assistant professor. Since 2010, he has been with AlcatelLucent (now Nokia), where he leads a research department at Nokia Bell Labs. His professional interest is related to the application of artificial intelligence to enable autonomous networking and design of mobile and wireless systems. He has $200+$ scientific publications (journals, conferences, and patent applications) and invited/tutorial talks. He is a Senior Member of the Institute of Electronics, Information, and Communication Engineering (IEICE).

Miao Liu (M'18) received the B.Sc. Degree in communication engineering from University of Electric Science and Technology of China, Cheng China in 2011, M.Sc degree, PhD degree in communication engineering from Southeast University, Nanjing, China and 2013 and 2019, respectively. $\mathrm{He}$ is assistant professor with Nanjing University of Posts and Telecommunications, Nanjing China. He has published more than 15 papers in IEEE international journal and conferences.

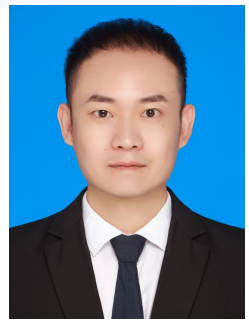

Jinlong Sun (M18) received the M.S. and Ph.D degrees from the Harbin Institute of Technology, Harbin, China, in 2014 and 2018, respectively. $\mathrm{He}$ is currently working as an Assistant Professor with the Nanjing University of Posts and Telecommunications, Nanjing, China. His current research interests include signal processing for wireless communications, machine learning, and integrated navigation systems.

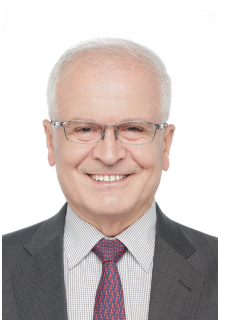

Hikmet Sari (F95) is currently Professor of Nanjing University of Posts and Telecommunications (NUPT), and also Chief Scientist of Sequans Communications. He received his Engineering Degree and $\mathrm{Ph} . \mathrm{D}$. from the ENST, Paris-Sud, Orsay. Prior to his current positions, he held various research and management positions in industry including Philips Research Laboratories, SAT, Alcatel, Pacific Broadband Communications, and Juniper Networks. His distinctions include the IEEE Fellow Grade (1995), the Andr Blondel Medal (also in 1995), the Edwin H. Armstrong Achievement Award in 2003, the Harold Sobol Award in 2012, as well as election to Academia Europaea (the Academy of Europe) and to the Science Academy of Turkey in 2012. Prof. Sari has served as an Editor of the IEEE Transactions on Communications (1987C1981), a Guest Editor of the European Transactions on Telecommunications (1993) and of the IEEE JSAC (1999), and an Associate Editor of the IEEE Communications Letters (1999C2002). He served as a Distinguished Lecturer of the IEEE Communications Society in $2001-2006$, as a member of the IEEE Fellow Evaluation Committee in 2002C2007, and as a member of the Awards Committee in 2005-2007. 


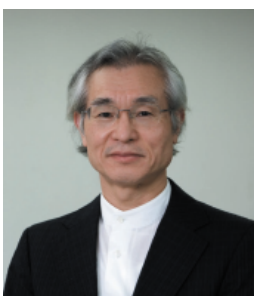

Fumiyuki Adachi (M'79-SM'90-F'02-LF'16) received the B.S. and Dr. Eng. degrees in electrical engineering from Tohoku University, Sendai, Japan, in 1973 and 1984, respectively. In April 1973, he joined NTT Laboratories and conducted various researches on digital cellular mobile communications. From July 1992 to December 1999, he was with NTT DoCoMo, where he led a research group on Wideband CDMA for 3G systems. In January 2000, he joined Tohoku University, Sendai, Japan, where he was a Professor at the Dept. of Communications Engineering, Graduate School of Engineering until he retired in March 2016. Currently, Prof. Adachi is a specially-appointed professor in the Research Organization of Electrical Communication at Tohoku University, and is continuing his research interest is in the area of wireless signal processing (multiaccess, equalization, antenna diversity, adaptive transmission, channel coding, etc.) and wireless networking. He is an IEICE Fellow and an IEEE Fellow. He is a recipient of the IEEE Vehicular Technology Society Avant Garde Award 2000, IEICE Achievement Award 2002, Thomson Scientific Research Front Award 2004, Ericsson Telecommunications Award 2008, Telecom System Technology Award 2009, Prime Minister Invention Award 2010, British Royal Academy of Engineering Distinguished Visiting Fellowship 2011, KDDI Foundation Excellent Research Award 2012, VTS Conference Chair Award 2014, C\&C Prize 2014, and IEEE VTS Stuart Meyer Memorial Award 2017, and IEEE ComSoc RCC Technical Recognition Award 2017. 